\title{
Convergencia entre formación \\ y extensión: intervención preprofesional \\ en Trabajo Social en la Universidad \\ Nacional de Córdoba
}

\author{
María Inés Peralta \\ mariainesperalta50@gmail.com
}

Sabrina Bermudez

sbermudez@arnet.com.ar

\section{Paola Garcia Elettore}

paola_garciaelettore@yahoo.com
Docentes investigadoras de la

Facultad de Ciencias Sociales,

Universidad Nacional de Córdoba,

Argentina.
Integración de la docencia y la extensión /

Intervenciones

RECEPCIÓN: 24/06/16

ACEPTACIÓN FINAL: 30/09/16

\section{Resumen}

Pensar las tres funciones de la universidad es un desafío que nos debe llevar a plantear prácticas de formación, investigación y extensión integrales, interrelacionadas e interdisciplinarias. El artículo aborda la experiencia concreta que estamos llevando adelante docentes de la asignatura Intervención preprofesional, que se dicta en el quinto nivel de la Licenciatura en Trabajo Social de la Facultad de Ciencias Social de la Universidad Nacional de Córdoba. Construir puentes entre el mundo académico y mundo profesional es una necesidad que requiere tanto de esfuerzos teórico-conceptuales como políticoinstitucionales. El primero se verá enriquecido al complejizarse los debates que definen los problemas de investigación y en el segundo se abrirán caminos para aportar a la necesaria autonomía de los profesionales respecto de las definiciones políticas de las instituciones en las que se desempeñan.

\section{Resumo}

Pensar nas três funções da universidade é um desafio que deve levar-nos a propor práticas de formação, pesquisa e extensão integrais, interligadas e interdisciplinares. $\mathrm{O}$ artigo aborda a experiência específica que os professores estão realizando os docentes da disciplina Intervenção Pré-profissional, que se ministra no quinto nível do Bacharelado em Serviço Social da Faculdade de Ciências Sociais da Universidade Nacional de Córdoba.

Construir pontes entre o mundo acadêmico e o profissional é uma necessidade que exige tanto esforços teórico-conceituais como político-institucionais. A primeira será enriquecido ao se complexificarem os debates que definem os problemas de pesquisa, e na segunda, abrir-se-ão caminhos para contribuir para a autonomia necessária dos profissionais a respeito das definições políticas das instituições em que trabalham.

Palavras-chave

- Treinamento

- Pesquisa

- Extensão

- Intervenção pré-profissiona
Para citación de este artículo Peralta, M. I.; Bermúdez, S. y García Elettore, P. (2016). Convergencia entre formación y extensión: intervención preprofesional en Trabajo Social en la Universidad Nacional de Córdoba. En Revista +E versión digital, (6), pp. 410-415. Santa Fe, Argentina: Ediciones UNL. 


\section{Universidad-sociedad como horizonte de sentido}

Si la relación universidad-sociedad es la totalidad de sentido desde la cual pensar las tres funciones de la universidad: docencia, extensión e investigación, producir conocimiento sobre las especificidades de cada una de ellas y la potencia que se despliega en las vinculaciones entre una/s y otra/s para fortalecer y ampliar aquella relación será un proceso-producto susceptible de abonar nuevas experiencias desde esta perspectiva.

Creemos que la relación universidad-sociedad no es una preocupación exclusiva de la extensión; afirmar lo contrario llevaría a pretender mirar la totalidad desde un centro privilegiado de sentido, y lo que nos proponemos justamente es mirar la totalidad desde la articulación de las diferencias entre las distintas funciones. ${ }^{1}$ Aunque sí es interesante resaltar la existencia de una mayor inclusión de precisiones sobre las funciones de docencia y de investigación en la letra de reglamentaciones, normativas y documentos del sistema universitario nacional en comparación con la función de extensión. ${ }^{2}$

Si bien no es objetivo de este trabajo profundizar en definiciones específicas sobre cada función, sí podemos vincular en términos generales a la docencia con la formación de recursos humanos, a la investigación con la producción de conocimientos y, a la extensión con la lectura de la demanda social, entendida esta última:

"como la expresión de una agenda, de temas y de perspectivas de tratamiento de los mismos en función de la correlación de fuerzas de sectores en un momento dado; y no como la demanda unilateral de un actor o como demanda de mercado. La construcción de esta agenda de la demanda social se realiza necesariamente en un proceso conformado de encuentro, escucha, debate y concertación con actores sociales, económicos y políticos del medio local del cual la universidad forma parte”. (Peralta, 2008a:1-2)

Lo que implica un posicionamiento desde la perspectiva del diálogo de saberes, la construcción histórico-social de la agenda pública y la interdisciplina.

\section{Las prácticas académicas pre profesionales en el campo societal}

Con este marco de referencia que pretende marcar la complejidad en la cual hay que construir los sentidos para desde allí codificar, interpretar, analizar, y elaborar conclusiones, pretendemos acercar la experiencia concreta que estamos llevando adelante desde la asignatura Intervención preprofesional, ubicada en el quinto nivel de la carrera Licenciatura en Trabajo Social perteneciente a la Facultad de Ciencias Social de la Universidad Nacional de Córdoba (UNC). Las prácticas preprofesionales están incorporadas a la formación desde el origen de esta profesión y persisten en el momento de su incorporación al sistema universitario nacional, más de 50 años atrás. Acevedo y Peralta proponen definirlas como:

"prácticas académicas, o sea como un espacio de enseñanza aprendizaje que se caracteriza por un contacto intencionado con la realidad con un objetivo de aprendizaje. A través de ellas, se espera que los estudiantes alcancen competencias de tres tipos: de conocimiento (lo que requiere implica poner en acto la teoría), procedimentales o aprendizajes del saber hacer (lo que implica la dimensión técnica) y actitudinales (lo que implica la dimensión ético-ideológica)". (2004:3)

Edelstein y Coria (1995), a través de la idea de "ritos de iniciación", desarrollan respuestas a la pregunta sobre qué es lo que hace de las prácticas de formación una instancia tan importante que deja sus marcas en la trayectoria futura, volviéndose un recuerdo casi ineludible, es decir, el peso que tienen estas prácticas en la trayectoria de formación de los estudiantes y futuros profesionales es estructurante.

Pero también nos interesa traer el aspecto urgente, amenazante, el sentido estratégico de las prácticas que señala Bourdieu (2007), producto de la improvisación e incertidumbre pero también de lo predecible y previsible. Esta característica debe ser explícitamente reconocida y valorada en el aprendizaje de lectura de lo social, e incorporada en las reflexiones teóricas que orientan las propuestas de las llamadas prácticas preprofesionales o académicas; ya que
1) Estatuto de la UNC "Artículo 2 - Misión de la Universidad. La Universidad, como institución rectora de los valores sustanciales de la sociedad y el pueblo a que pertenece, tiene los siguientes fines:

a) La educación plena de la persona humana.

b) La formación profesional y técnica, la promoción de la investigación científica, el elevado y libre desarrollo de la cultura y la efectiva integración del hombre en su comunidad, dentro de un régimen de autonomía y de convivencia democrática entre profesores, estudiantes y graduados. c) La difusión del saber superior entre todas las capas de la población mediante adecuados programas de extensión cultural.

d) Promover la actuación del universitario en el seno del pueblo al que pertenece, destacando su sensibilidad para los problemas de su época y las soluciones de los mismos.

e) Proyectar su atención permanente sobre los grandes problemas y necesidades de la vida nacional, colaborando desinteresadamente en su esclarecimiento y solución". 2) Sólo como ejemplo, vale señalar que en documentos actuales - año 2006que se utilizan para la acreditación de carreras, se definen con precisión y llamadas al pié, las funciones de docencia y de investigación, pero la función de extensión sólo es nombrada. O también la reciente y básica inclusión de indicadores de la función de extensión en las pautas de distribución presupuestaria del CIN. 
lejos estaría esta característica de ser un problema para la docencia o de ser un condicionante que perturba los objetivos de aprendizaje y altera los ritmos y cronogramas académicos. Por el contrario, recuperar la improvisación e incertidumbre como contexto de los problemas a los cuales el saber y el saber hacer deben dar respuestas, plantea desafíos a los perfiles profesionales de diversas disciplinas.

Entonces entendemos que "las prácticas son estructurantes y, así como la práctica social estructura el habitus, la práctica preprofesional estructura el habitus disciplinar" (Peralta, 2012:18). Las prácticas de intervención preprofesional del V nivel de la carrera de Trabajo Social pretenden que en un espacio social particular (institucional y/o comunitario) se diseñe, desarrolle y sistematice una estrategia de intervención fundada que recupere aprendizajes teórico-metodológicos adquiridos durante la formación previa y que se constituya en objeto de reflexión y fundamentación teórica desde el campo de la teoría social (Plan de estudios 2004). El desarrollo de la asignatura (y de todo el Plan de estudios) se enmarca en un contexto universitario y también municipal, provincial y nacional. Situados en un escenario de profundos cambios a nivel latinoamericano, asistiendo a un conjunto de políticas públicas de corte neoliberal que atraviesa las estructuras sociopolíticaeconómica, cultural y educativa. En este contexto, los desafíos se complejizan tanto para la formación de los estudiantes como en los espacios donde desarrollan sus prácticas de formación. Desde este espacio la cátedra apunta a construir con los estudiantes y los referentes institucionales de los distintos centros de prácticas, intervenciones fundadas desde los derechos sociales. Entendiendo que las universidades tienen responsabilidad en la formación de personas que trabajen por el cumplimiento más amplio y abarcativo de los derechos humanos, acreditando no sólo profesionales de excelencia sino agentes que actúen desde un proyecto ético político tendiente a construir mayores posibilidades de libertad, justicia e igualdad.

El análisis de las prácticas preprofesionales se ubica en dos dimensiones:

1) La incidencia de prácticas y producciones en los procesos de formación y en la construcción/cementación del campo profesional (dimensión endógena).

2) La ubicación de las prácticas preprofesionales en el contexto societal tanto científico/universitario como el económico/político/ social/cultural (dimensión exógena).

Estas prácticas se dan en un campo de disputas, de conflictos, observamos a la currícula como un campo de conflictos de capitales, a los centros de prácticas como campos de disputa donde la profesión se encuentra en tensión entre espacio profesional y espacio ocupacional (políticas sociales). La tensión en el campo estatal sobre la naturaleza de las políticas sociales y la incorporación/ampliación de la mirada desde los derechos. Todo en una sociedad que disputa capitales y reconocimiento de necesidades y derechos. Finalmente, decimos que las prácticas preprofesionales están atravesadas por conflictos pedagógicos, sociales y políticos. En este campo de conflictos se desarrollan las prácticas de formación profesional que definimos como construcción de prioridades sociales, ya que al decir de Crosetto, Sueldo y García:

"ocupan espacios de debate, intercambios, confrontación de sentidos y disputas casi de manera constitutiva al desarrollo de la profesión y, nos interpelan permanentemente a los diferentes actores involucrados en el ámbito académico (estudiantes, docentes, graduados, no docentes) como en el gremial (instituciones público estatales y societales, centros de práctica, referentes institucionales, entre otros)". (2016:7)

\section{La naturaleza extensionista de la intervención preprofesional}

La intención de este trabajo es hacer un aporte a la integración de las funciones de docencia, investigación y extensión, para lo cual resulta necesario explicitar la noción de totalidad desde la cual nos paramos. Noción fundamental para pensar la relación entre las partes y el todo. Al respecto, hay que llamar la atención acerca de entender la totalidad como algo abierto y en proceso de constitución, de ningún modo como cerrada y absoluta. De ese modo, admite una comprensión de la realidad en la que siempre hay posibilidad de una nueva pregunta y de un vacío por reconocer. Sin lugar a dudas requiere un gran esfuerzo mirar así la realidad, ya que predominan los tratamientos binarios basados en la idea de opuestos preexistentes, con autonomía y como entidades independientes.

Desde esta noción de totalidad, las funciones de docencia, investigación y extensión son un hecho educativo, de diálogo con el otro y de descubrimiento, que se materializa en la propuesta que los docentes desarrollamos para la asignatura.

La práctica de intervención preprofesional se diferencia de las de años anteriores de cursado por el tiempo de permanencia en la institución, que comienza con los primeros acuerdos a finales del cuarto año y concluye un año después con la finalización del quinto año.

Este espacio temporal permite procesos de inserción y acuerdos para la implementación de planes de trabajos que se entroncan con los programas y las acciones institucionales. De este encuadre, se puede visualizar la incorporación efectiva a los equipos técnicos institucionales, en los que los/as estudiantes son incluidos en instancias de diagnóstico, planificación, ejecución y evaluación de las intervenciones realizadas institucionalmente. Algunas instituciones facilitan la participación en ateneos de formación, 


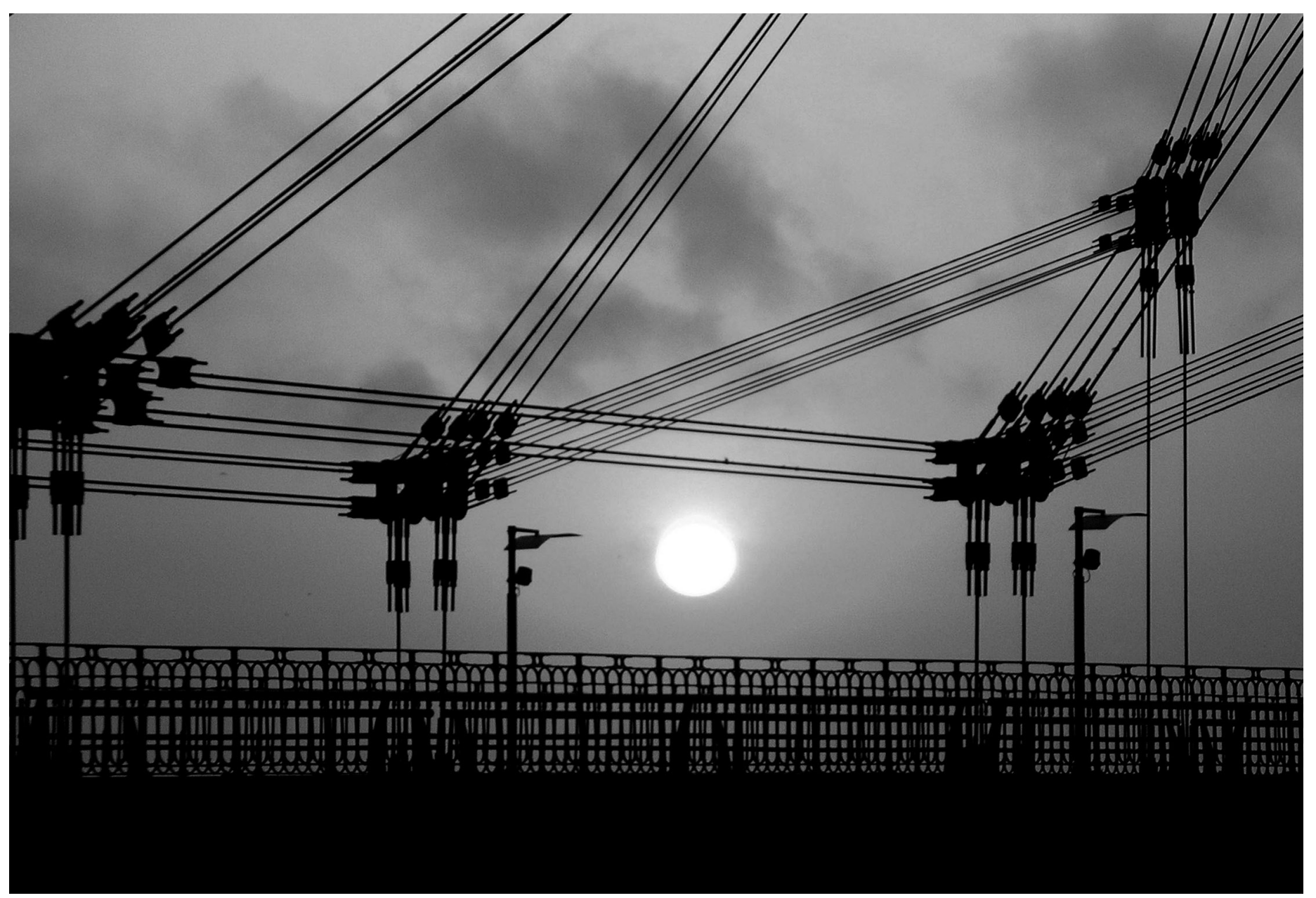

(๑) Laura Hormaeche

\section{G6}

las prácticas académicas constituyen un entramado complejo de preocupaciones, tensiones y contradicciones que requieren del debate multiactoral permanente en el proceso de formación 
cursos de capacitación, seminarios, los cuales se constituyen en instancias complementarias a la formación académica. También podemos identificar que este diseño académico se asemeja a un proceso de "formación en servicio", puesto que las prácticas de intervención preprofesional contienen componentes de transferencia y de ejecución efectiva de acciones que refuerzan, amplían e interpelan las prácticas institucionales (UNC, Programa de la asignatura 2015/16).

En el proceso de enseñanza-aprendizaje, una de las figuras centrales resultan los referentes institucionales de los centros de prácticas

"profesionales de Trabajo Social, otros profesionales, actores sociales y políticos con trayectorias en el área específica y en problemáticas particulares que acompañan el proceso de enseñanza-aprendizaje de los estudiantes; asumiendo un rol significativo en la definición y orientación de las actividades de terreno" (UNC. Res. HCACETS 159/2014).

De acuerdo con la misma resolución, las funciones de los referentes institucionales son la de elaborar y coordinar de manera articulada con el docente un plan de trabajo, acompañar y efectuar el seguimiento en las acciones que desarrollen los estudiantes, orientándolos. De la interacción de la triada conformada por estudiantes, docentes y referentes institucionales se generan las condiciones para un proceso de diseño e implementación de la estrategia que a lo largo de un año se llevará adelante para contribuir a la transformación de una situación problemática, generando para ello instancias de trabajo continuo de los estudiantes en los centros de prácticas/instituciones, de construcción de consensos y condiciones institucionales.

La asignatura intervención preprofesional propone la construcción de un diseño de estrategia de intervención producto de la intersección de dos campos: la universidad (estudiantes desarrollando las prácticas y docentes como responsable pedagógico de ese proceso) y la institución o el territorio: donde referentes de la institución guían las practicas de los estudiantes y en una relación dialéctica de transferencia de conocimientos construyen la demanda y la estrategia que los estudiantes desarrollarán en conjunto con los agentes institucionales. En este proceso se ponen en juego los conocimientos científicos y los que circulan en el campo societal; se acercan, diseñan, implementan y evalúan acciones que desde el campo de las ciencias sociales aportan a las problemáticas que se tradujeron en demandas sociales. Se destaca que el diseño e implementación de estrategias requieren de una búsqueda y construcción de argumentos teóricos que demandan a los estudiantes la vinculación con investigadores e investigaciones desarrolladas en nuestra carrera para complementar los marcos de referencia construidos, lo que da una significancia local a las problemáticas sociales que se abordan. En estos espacios pedagógicos se construye conocimiento desde la empiria en diálogo con las teorías sociales con el fin de la reconstrucción colectiva de un pensamiento crítico.

Las prácticas académicas constituyen un entramado complejo de preocupaciones, tensiones y contradicciones que requieren del debate multiactoral permanente en el proceso de formación, ahí radica su carácter integrador y la puesta en juego de las funciones de docencia, investigación y extensión. Si pensamos que la extensión en general es definida como los aportes que la universidad realiza a la sociedad desde una visión articuladora, podemos visualizar el carácter extensionistas de la asignatura no sólo desde la perspectiva constructivista que proponemos, sino desde lo cuantitativo. Anualmente, en promedio 40 grupos de alumnos trabajan en diferentes instituciones públicas estatales y de la sociedad civil, construyen lecturas de escenarios de intervención, análisis de los sujetos de intervención, objetos de intervención y desarrollo de estrategias que tiendan a transformar, mejorar las condiciones materiales y simbólicas de vida de los sujetos.

Finalizadas las prácticas, los estudiantes escriben una tesina sobre ese proceso, lo que implica cierta construcción de conocimiento que en términos de aportes nutre a la institución/centro de práctica, al campo profesional, a los sujetos, a las políticas públicas y a la universidad. En este sentido, la presencia e interacción académica que se produce con las organizaciones y la complejidad e integralidad de la propuesta hacen necesario el desarrollo de las funciones de docencia, investigación y extensión en estos procesos de trabajo.

El proceso de enseñanza-aprendizaje es el resultante de un complejo entramado de acciones donde los actores van delimitando el camino a transitar a partir de consensos y de una vinculación basada en la participación protagónica para la transformación. Nos proponemos generar un espacio colectivo de producción, que estimule la búsqueda, la pregunta, la problematización, es decir, un espacio caracterizado por la circulación y respeto de la palabra, la deconstrucción de lo viejo, la iniciativa de construir lo nuevo, la implementación de dispositivos para la comprensión de lo que sucede y para pensar en los cambios, la posibilidad de pensarse como sujetos políticos de transformación. Un espacio de trabajo donde todos participan y protagonizan la búsqueda, elaboración, intercambio, circulación y colectivización de conocimientos para la transformación de la realidad social.

El proceso pedagógico se basa en la orientación, supervisión e interpelación dialógica del equipo docente con el grupo de estudiantes y los referentes institucionales a fin de acompañar el proceso de intervención preprofesional desde una lógica de investigación-acción, que recupere los contextos institucionales y comunitarios, los contextos históricos, sociales, políticos, 
económicos y culturales, así como fundamentos teóricos acerca de la problemática, los sujetos, las políticas públicas en torno al problema objeto y la realización o ejecución de acciones o acompañamiento a las actividades que lleva adelante la institución, lo que permite trabajar desde un marco institucional con cierta autonomía que dé lugar a la mirada crítica y propositiva en torno a puntos de tensión o nudos críticos que puedan aparecer. La práctica es un proceso complejo que requiere de flexibilidad, adaptación y creatividad permanente para ser permeable a la realidad social, cultural, política, e institucional y/ o comunitaria que le da marco. El conocimiento producido en las instancias de docencia e investigación-acción encuentra su objetivo en la función de extensión. Las experiencias desarrolladas a lo largo de estos años ponen de manifiesto la intencionalidad de las prácticas de intervención preprofesional de poner el acento en la producción y construcción de conocimiento científico al servicio de la sociedad y la mejora en la calidad de vida de los ciudadanos en el encuentro con la realidad social misma y los actores que en ella intervienen. Estas prácticas constituyen aportes para la transformación de problemas sociales y condiciones de opresión y aportan a la construcción de una sociedad justa, solidaria, fraterna y libre.

\section{A modo de cierre}

Son muchas las posibilidades a desarrollar para articular desde perspectivas integrales las funciones de docencia, investigación y extensión. Requieren de espacios dialógicos que potencien y amplíen los sentidos y espacios de articulación, que potencien y amplíen la acción. Las modalidades que puede tomar la integración son diversas, como también son diversos los impactos positivos en estas experiencias de integración. Dinamitar las fracturas profundas entre el mundo académico y el mundo profesional supone una necesidad de respuestas teórico-conceptuales y políticoinstitucionales. Las primeras se verán enriquecidas al complejizarse los debates que conducen a la definición de los problemas de investigación y las segundas abrirán caminos para aportar a la autonomía de profesional respecto de las definiciones políticas de las instituciones $\mathrm{y} / \mathrm{u}$ organizaciones.

Proponer un programa que intente poner en juego en el campo de la formación, investigación y extensión la resolución de problemas reales, concretos, sentidos, desde escenarios institucionales o territoriales complejos, dialogando con los conocimientos de las ciencias sociales y con la pluralidad de actores profesionales y sociales, implica además una problematización constante de las prácticas de enseñanza, de las formas de entender el aprendizaje y de evaluación para que los estudiantes logren su acreditación en procesos integrales.

\section{Referencias bibliográficas}

Acevedo, P. y Peralta, M. I. (2004). Aportes al debate en torno a las prácticas académicas y formación profesional en Trabajo Social. Ponencia. Encuentro Académico Nacional de FAUATS: "Formación académica y procesos de reforma curricular en las carreras de Trabajo Social". Luján.

Bourdieu, P. (2007). El sentido práctico. Buenos Aires: Siglo XXI Editores.

Crosetto, R.; Sueldo, J. y García, P. (2016). Experiencias de prácticas académicas. Visibilizando nuestro saber-hacer. Cuadernos de trabajo, Serie Docencia. Escuela de Trabajo Social, UNC. Córdoba.

Edelstein, G. y Coria, A. (1995). Imágenes e imaginación. Iniciación a la docencia. Buenos Aires: Kapelusz.

Parisi, A.; Peralta, M.I. y otros (2008-2009). Documentos de investigación: "La cuestión de la diferencia en las ciencias sociales: género, educación y política”. ETS-UNC-SECYT. Mimeo.

Peralta, M. I. (2008a). Aportes al debate necesario sobre la extensión universitaria en la universidad pública latinoamericana actual. Ponencia. Encuentro Latinoamericano Extensión Universitaria. Mar del Plata.

Peralta, M. I. (2008b). Pronunciamiento sobre la función de Extensión en la Universidad Pública. II Foro de Extensión Universitaria - UNC, 2 y 3 de octubre. Recuperado de: http://www.unc.edu.ar/novedades/2008/noviembre/ii_foro_de_ extension_unc.pdf

Peralta, M. I. (2012). Diseño de estrategias de intervención. En Soldevila, A. (Coord.), Práctica de intervención preprofesional: construcción de procesos de conocimiento. Aportes Educativos. Córdoba: Escuela de Trabajo Social, UNC. Rozas Pagaza, M. (2005). Tendencias teórico-epistemológicas y metodológicas en la formación profesional. En Molina, M.L., La cuestión social y la formación profesional en trabajo social en el contexto de las nuevas relaciones de poder y la diversidad latinoamericana. Buenos Aires: Espacio Editorial-ALAETS.

Soldevila, A. (Coord.). Práctica de intervención preprofesional: construcción de procesos de conocimiento. Aportes Educativos. Córdoba: Escuela de Trabajo Social, UNC.

Sousa Santos, B. de (2006). El Milenio Huérfano. Ensayos para una nueva cultura politica. Madrid: Trotta.

Universidad Nacional de Córdoba. Programa Intervención Preprofesional 2015/2016. Recuperado de: http://trabajosocial.unc.edu.ar/?q=programas-asignaturas (29/05/2016).

Universidad Nacional de Córdoba. Res. HCACETS 159/2014. 